\title{
Même chez les médecins, la publicité est efficace!*
}

Urs Strebel ${ }^{a}$,

Alain Michaud ${ }^{b}$

a Dr méd., ex médecin-chef du département de médecine interne, Spital Männedorf

b Dr méd., médecine générale FMH, Nyon, Chargé de Cours à l'Institut Universitaire de Médecine Générale (IUMG) de l’Université de Lausanne.
L'impact de la publicité une expérience personnelle

Déjà pendant mes études (mon diplôme fédéral date de 1972) et plus encore pendant ma formation postgraduée, j'étais régulièrement contacté par des représentants de l'industrie pharmaceutique qui souhaitaient m'informer au sujet de leurs produits et me remettre un petit cadeau ou une profusion d'échantillons. Je me souviens très bien de l'introduction du Valoron, vanté comme un antalgique exceptionnel. Grâce à son action, soi-disant différente de celle des opiacés traditionnels, il n'avait pas été inscrit sur la liste des stupéfiants (LStup) et il a été de ce fait largement distribué. N'empêche qu'un ou deux ans plus tard, le Valoron figurait sur la liste de la LStup.

Je n'avais à cette époque pas l'impression d'être influencé, et je ressentais même un lien, presque une confraternité, avec les représentants de l'industrie pharmaceutique. II s'agissait de personnes sympathiques et serviables, que je ne voulais pas offusquer lorsqu'ils venaient me solliciter. II arriva qu'un jour, je n'eus pas eu le temps de recevoir un représentant; il me téléphona alors pour $\mathrm{m}^{\prime}$ expliquer qu'il avait une famille et qu'il devait justifier d'un certain nombre de contacts avec des médecins pour percevoir la totalité de son salaire. Il va de soi que je lui accordai alors un rendez-vous. Plus tard, alors que j'étais chef de clinique à l'Hôpital universitaire de Zurich, mon chef me proposa de participer à une formation continue à New York, ce que j'acceptai avec plaisir. Elle n'avait pas

\section{Premier constat: L'industrie consacre beaucoup d'argent à la publicité}

En janvier 2000, Ashley Wazana publiait dans le JAMA un article intitule «Physicians and the pharmaceutical industry: Is a gift ever just a gift?» [1]. Il y constatait que (a) l'industrie pharmaceutique aux USA dépense entre 8000 et 13000 US-\$ par année et par médecin en marketing, (b) les interactions entre le corps médical et l'industrie revêtent des formes très diverses (formations pré-, postgraduée et continue, cadeaux, échantillons, repas, participation à des congrès - frais de déplacement inclus, etc.) et (c) les médecins n'ont pas une conscience assez claire de leur propre influençabilité. été financée par un pool, mais par une entreprise pharmaceutique. Mais il me fut par la suite impossible de décliner les offres de la représentante responsable (une dame d'ailleurs fort sympathique et séduisante). Ces offres ne concernaient encore que des entretiens, au cours desquels elle évoquait pourtant très souvent les avantages d'un médicament, alors que nous avions le produit concurrent en stock. Un an après, un voyage à Boston m'était offert. Boston était intéressant et valait la peine d'être vu. Et encore un an après (en 1988), le produit en question, sur lequel j'avais été parfaitement renseigné, figurait dans notre stock. Même si, sur la base des documents que j'avais obtenus, je pouvais honnêtement me prononcer en faveur de cette nouvelle substance, je ne me sentais pas aussi libre que je l'aurais souhaité dans mon argumentation devant la commission des médicaments.

A partir de cette expérience, j'ai décidé de ne plus me baser que sur la littérature - et non plus sur les dires des représentants de l'industrie pharmaceutique - pour $m^{\prime}$ informer au sujet des nouveaux médicaments. Depuis lors, la question de la collaboration entre le corps médical et l'industrie m'a préoccupé et c'est pour cette raison que je me suis engagé dans l'élaboration des directives de I'Académie Suisse des Sciences Médicales (ASSM). Et cette collaboration est également la raison de ma participation à la commission consultative de I'ASSM.

Urs Strebel

Dans son éditorial «The pharmaceutical industry: To whom is it acountable?» [2], également publié en 2000, Marcia Angell critique la disproportion entre le budget accordé à la recherche et celui investi dans la publicité aux USA. Il existe aussi des évaluations pour l'Europe (malheureusement, l'industrie pharmaceutique ne dévoile pas de chiffres exacts): en 2000, l'industrie allemande consacrerait $30 \%$ de son chiffre d'affaires à la publicité (sponsoring inclus), ce qui représenterait plus du double des dépenses consacrées à la recherche [3]. Novartis et Roche révèlent des chiffres analogues [4]. On peut dès lors supposer que l'industrie pharmaceutique sait exactement dans quel domaine le retour sur l'investissement est le plus élevé. 


\section{Deuxième constat: Les médecins n'ont pas conscience de leur influençabilité}

La prise de conscience de cette réalité semble, en revanche, bien moins nette au sein du corps médical. Lorsque l'on discute entre confrères des directives de l'ASSM «Collaboration corps médical-industrie», publiées en 2005, l'enthousiasme est tout à fait modéré: non seulement un grand nombre de médecins ne peuvent s'imaginer une formation continue sans sponsors, mais encore la plupart d'entre eux nient être influençables. Le bien-être des patients serait, à les entendre, tellement prioritaire qu'ils se sentiraient à l'abri de toute mauvaise influence.

Cette réaction est universelle et c'est l'une des raisons pour laquelle l'ASSM a créé en 2007 un poste de consultation et une commission consultative pour la mise en œuvre des directives [5], qui a publié un premier rapport [6] en 2008.

Kerry Breen résume la problématique dans son article «The medical profession and the pharmaceutical industry: when will we open our eyes?»[7], publié en 2004 dans le Medical Journal of Australia, et énumère six items, qu'il justifie avec des références littéraires et résume comme suit:

- La plupart des médecins partent de l'idée que les cadeaux n'influencent pas leurs habitudes de prescription;

- Le nombre de cadeaux reçus est inversément proportionnel à l'avis des médecins selon lequel l'accueil de délégués médicaux n'a aucune influence sur les prescriptions;

- 80 à 95\% des médecins accueillent régulièrement des représentants de l'industrie pharmaceutique;

- Plus les contacts avec des représentants sont fréquents, plus les médecins font des prescriptions inutiles et prescrivent de nouveaux médicaments;

- Les prescriptions des produits présentés dans les formations continues sponsorisées sont en nette augmentation durant les six mois qui suivent la formation;

- Selon les estimations, l'industrie pharmaceutique dépense environ 21000 dollars australiens par année et par médecin pour la publicité.

La sous-estimation de la part des médecins de l'influence qu'ils subissent lors des visites de délégués médicaux est encore plus patente quand on les interroge sur la perception qu'ils en ont: Selon une statistique de Steinmann et al. datant de 2001 [8], seuls 1\% des médecins se disent «très influencés»; $38 \%$ d'entre eux pensent être «faiblement influencés» et $61 \%$ «pas influencés du tout». Mais il en va tout autrement lorsque ces mêmes médecins sont appelés à juger leurs collègues: cette fois, $33 \%$ d'entre eux seraient très influencés, $51 \%$ faiblement influencés et $16 \%$ pas influencés du tout.

\section{Utilisation par l'industrie de la règle de la réciprocité}

Certains médecins et sociétés de discipline médicale ont tiré les conséquences de ces faits. Afin d'éviter les conflits d'intérêts, l'American Psychiatric Association (APA) a déclaré le 25 mars 2009 dans un communiqué qu'à l'avenir elle n'organisera plus de manifestations sponsorisées [9]. Les psychiatres connaissent la règle de la réciprocité. Elle joue un rôle important dans la psychologie de la publicité et nous apparaît comme évidente.

Selon cette règle, l'être humain est prêt à offrir une contrepartie dès qu'il obtient quelque chose; autrement dit, lorsqu'il reçoit un cadeau, il se sent obligé d'en quelque sorte rembourser le donateur en lui fournissant une prestation compensatoire (Wikipedia). Dans les affaires et en milieu francophone, on parle du principe du «retour d'ascenseur»..

On peut mentionner dans ce contexte le travail récemment publié de David Grande [10]: «Effect of exposure to small pharmaceutical promotional items on treatment preferences». Il s'agit dans cet article de l'influence des petits cadeaux sur les habitudes de prescriptions des médecins; la moitié d'entre eux s'étant formés à l'Université de Pennsylvania School of Medicine, où ils ont étudié la problématique des relations entre le corps médical et l'industrie, et l'autre moitié à la Miami Miller School of Medicine où ce sujet n'a pas été traité. Comme il fallait s'y attendre, les étudiants ont été influencés par les cadeaux; toutefois ceux de Pennsylvannie se sont révélés moins influençables que leurs collègues de Floride.

L'industrie fait jouer la règle de la réciprocité en sachant parfaitement ce qu'elle fait! On peut aisément supposer que cette règle du business est efficace dans la relation corps médical-industrie et qu'elle est payante, sinon les firmes pharmaceutiques - essentiellement orientée vers le gain - auraient depuis longtemps changé de stratégie. En 2004, Blumenthal a publié à ce sujet un résumé digne d'être lu, «Doctors and Drug Companies», qui éclaire bien cet aspect des choses [11].

Nous renonçons délibérément à parler plus en détails de la publicité dans les journaux médicaux qui, à l'évidence, par le martèlement auquel elle nous soumet, est destiné à influencer nos habitudes de prescriptions.

\section{Alors ... que faire?}

Dans le NEJM du 21 mai 2009, Robert Steinbrook [12] résume les dernières recommandations de l'Institute of Medicine à propos des relations entre le corps médical et l'industrie: (a) toutes les institutions médicales devraient élaborer des directives concernant les gestions des conflits d'intérêts, (b) le Congrès est invité à définir des règles. Des paragraphes spéciaux sont consacrés (c) à la recherche et aux formations pré- et postgraduées et continue, qui devraient être libres de 
toute influence de l'industrie. Il est également recommandé (d) de n'accepter aucun cadeau et (e) de limiter les relations avec les représentants de l'industrie pharmaceutique au strict minimum.

Si nous, médecins, ne voulons pas nous laisser manipuler, nous devons - tant à titre individuel que collectif - prendre conscience des mécanismes de l'influençabilité et en tirer les conséquences. Nous devrions être en mesure de choisir librement les médicaments ainsi que les contenus et la forme des sessions de formations continues. Au bout du compte, cela pourrait signifier pour les médecins: (1) qu'ils renoncent à accueillir des délégués médicaux, (2) qu'ils acquièrent leur savoir dans la littérature spécialisée indépendante et (3) qu'ils renoncent à participer à des formations sponsorisées.

Au bout du compte, disions-nous ... il faut toutefois rester conscients des conséquences qu'auraient ces mesures. La première mesure pourrait, par exemple, mettre au chômage quelques centaines de délégués médicaux; quant à la troisième mesure, elle mettrait clairement en péril l'existence même d'un certain nombre de congrès médicaux, dont le contenu est pourtant souvent d'un très haut niveau.

Contrairement aux médecins d'hôpitaux, les praticiens indépendants ne perçoivent pas de revenus lorsqu'ils s'absentent de leur cabinet, alors que les frais continuent à courir en leur absence; ils estiment alors - à tort ou à raison - que la perte financière ainsi engendrée justifie que ledit congrès ne coûte pas trop cher. Ces réflexions ne sont probablement pas tout à faits exactes (10 jours de formation continue sont inclus dans TARMED), cependant elles existent et doivent dès lors être connues.

Il serait intéressant d'évaluer la baisse du prix des médicaments susceptible de résulter d'une part d'une moindre participation des industries pharmaceutiques - voire de leur absence totale - aux congrès et autres manifestations de formation continue et, d'autre part, de la cessation des visites de délégués médicaux dans les cabinets et les hôpitaux. On pourrait alors tenter de persuader les politiciens et les assureurs de redistribuer ce bénéfice aux médecins sous forme de subvention horaire à la formation continue, comme le font déjà certains pays limitrophes. Toutefois, il y a loin de la coupe aux lèvres, et nous craignons que ce type de propositions novatrices ne fasse pas recette et qu'en fait de coupe, nous restions sur notre soif!

Dans ces conditions, comment doit se comporter le médecin? De nombreux articles - nous n'en avons cité qu'une part infime - devraient nous persuader qu'aucun d'entre nous n'échappe à la règle de la réciprocité évoquée plus haut. De ce fait, l'ASSM a édicté des directives «Collaboration corps médical - industrie». Mais, même si celles-ci existent depuis cinq ans déjà, leur application laisse malheureusement à désirer; chacun de nous devrait se sentir concerné et s'efforcer de les mettre en pratique.

Mais surtout ... les médecins, à titre individuel, devraient faire preuve d'une salutaire prise de conscience et, au minimum, se faire un devoir éthique de refuser un certain nombre de cadeaux et/ou de renoncer à participer à certaines manifestations où «la ficelle publicitaire est trop grosse», c'est-à-dire quand la tentative de prise de pouvoir de l'industrie est grossièrement évidente. Comme le disait dans notre commission le représentant des firmes pharmaceutiques, ce n'est pas forcément à elles de renoncer à nous tenter, c'est surtout à nous d'apprendre à dire clairement non. Il en va de notre éthique et, osons le mot, de notre honneur.

\section{Références}

1 Wazana A. Physicians and the pharmaceutical industry: Is a gift ever just a gift? JAMA. 2000;283: 373.

2 Angell M. Is academic medicine for sale? NEJM. 2000;342:1516.

3 Deutsches Ärzteblatt. 2001;98:A 2484.

4 NZZ. 28. Mai 2002.

5 Reinhart W. Collaboration corps médical-industrie. Poste de consultation et commission consultative de l'ASSM. Bull Méd Suisses. 2007;88(33):1346.

6 Amstad H, Reinhart W. Rapport d'activités du poste de consultation et de la commission consultative pour la mise en pratique des directives «Collaboration corps médical-industrie». «Je m'inscris au cours de formation continue. Je suis végétarien.» Bull Méd Suisses. 2008;98(35):1490.

7 Breen K. The medical profession and the pharmaceutical industry: when will we open our eyes? Med J of Australia. 2004;180:409.

8 Steinmann $\mathrm{M}$ et al. Of principles and pens: attitudes and practices of medicine housestaff toward pharmaceutical industry promotions. Am J Med. 2001;110:551.

9 American Psychiatric Association. The AMA phases out industry supported symposia, News Release. 2009; March 25. (www.psych.org).

10 Grande D. et al. Effect of exposure to small pharmaceutical promotional items on treatment preferences. Arch Intern Med. 2009;169:887.

11 Blumenthal D. Doctors and drug companies. NEJM. 2004;351;1885.

12 Steinbrook R. Controlling conflict of interest proposals from the institute of medicine. NEJM. 2009;360:2160. 